\title{
Un titre de spécialiste pour gérer les tâches administratives?
}

\author{
Jürg Unger-Köppel \\ Dr méd., membre du Comité central de la FMH, responsable du département Médecine et tarifs hospitaliers
}

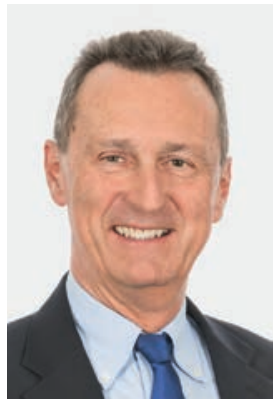

L’ASMAC le répète depuis des années et notre enquête avec gfs.bern ${ }^{1}$ le confirme depuis sa première édition: les tâches administratives à la charge des jeunes médecins ne cessent d'augmenter. Cela pourrait donner l'impression que la formation de spécialiste devrait plutôt permettre de relever avec succès les défis bureaucratiques que d'offrir une bonne médecine aux patients. Avec de telles perspectives, personne n'a envie de se lancer sur la voie ardue des études en médecine humaine. Au terme de leur scolarité, les jeunes rêvent bien plus de travail avec et pour les patients, et ils ont bien raison car c'est ce qui rend notre profession si attrayante.

En même temps, une pénurie de relève pèse sur les hôpitaux. La situation est généralement un peu moins accentuée en agglomération que dans les zones plus rurales. Là, il n'est pas rare d'entendre: «On a du mal à trouver des médecins et du personnel infirmier.» Pour les hôpitaux confrontés aux problèmes de relève, la fermeture est une option abordée très rapidement mais son impossibilité politique est tout aussi rapidement décriée. Dans cette situation, il serait préférable de penser à l'avenir des hôpitaux plutôt que de parler de leur simple fermeture. Laisser une infrastructure coûteuse se détériorer, à l'instar des installations militaires bâties sur des zones qui ne présentent désormais plus aucune menace, ne peut pas être une solution économiquement viable. Ce qu'il faut, ce sont de nouvelles structures qui apportent des réponses pertinentes aux évolutions du secteur de la santé et des solutions pragmatiques. Comment appliquer les possibilités de la télémédecine, la collaboration interprofessionnelle ou le nombre minimum de cas exigés? Quelles structures disposent de possibilités de transport modernes dans les cinq à sept régions hospitalières dont tout le monde parle et comment transformer ces solutions en un système général efficace?

Précisément avec des objectifs redéfinis, les sites périphériques plus développés d'une région hospitalière ont besoin de médecins bien formés et capables de faire face à leurs nouvelles tâches. Aujourd'hui, les jeunes médecins ont de plus en plus tendance à rester dans les agglomérations pour satisfaire aux exigences de leur couple, à savoir que les deux puissent se réaliser professionnellement. Que ferait un universitaire non-médecin dans une région reculée de Suisse qui n'est pas en mesure de lui proposer un emploi? Si un couple ne veut pas être réduit à ne se voir que le weekend, il aura vite pris la décision de rester en agglomération. Pour ces régions reculées, cela signifie donc de fournir des efforts particuliers pour développer l'attractivité de leur marché de l'emploi.

Les bons formateurs exercent une force d'attraction cruciale, presque magnétique, sur les jeunes médecins. En même temps, les hôpitaux qui réussissent à recruter sont justement ceux qui s'organisent pour que les médecins en formation travaillent davantage avec les patients et doivent moins s'occuper des tâches administratives. De plus, confier une part élevée de tâches administratives aux médecins n'est pas rentable pour les hôpitaux. Rien qu'un léger transfert de ces tâches des médecins et des infirmières vers des assistants administratifs présente un fort potentiel d'économie. Les hôpitaux qui, conjointement avec leur personnel, recherchent activement des moyens de ramener les médecins au chevet de leurs patients servent plusieurs objectifs à la fois: augmenter la satisfaction au sein du corps médical et accroître l'attractivité des postes de travail. De plus, ils économisent des coûts parce que les médecins exercent de manière ciblée des activités que personne d'autre ne pourrait accomplir à leur place. Ils arrivent à ce que les tâches d'un centre hospitalier soient réalisées avec moins de médecins tout en augmentant, et c'est sans doute le plus important, la satisfaction des patients, car le patient voit son médecin plus souvent.

Par conséquent, les conseils d'administration des hôpitaux seraient certainement très inspirés de donner le mandat pour que tout soit entrepris dans leur institution afin de recevoir la Rose d'hôpital décernée par l'ASMAC (cf. l'article sur l'attribution de cette distinction à la page 36). 